Research Article

\title{
Network Pharmacology-Based Identification of the Mechanisms of Shen-Qi Compound Formula in Treating Diabetes Mellitus
}

\author{
Zhipeng Hu $\mathbb{D}^{1},{ }^{1}$ Maoyi Yang $\mathbb{D}^{1},{ }^{1}$ Liangjun Yang, ${ }^{2}$ Chunguang Xie $\mathbb{D}^{1},{ }^{1}$ Hong Gao, \\ Xiaoxu Fu, ${ }^{1}$ Hongyan Xie, ${ }^{1}$ and Ya Liu ${ }^{1}$ \\ ${ }^{1}$ Hospital of Chengdu University of Traditional Chinese Medicine, Chengdu 610072, China \\ ${ }^{2}$ Department of Gastroenterology, Tongde Hospital of Zhejiang Province, Hangzhou 310012, China
}

Correspondence should be addressed to Chunguang Xie; xiecg@cdutcm.edu.cn

Received 21 October 2019; Revised 20 February 2020; Accepted 9 April 2020; Published 4 June 2020

Academic Editor: XiuMin Li

Copyright (c) 2020 Zhipeng Hu et al. This is an open access article distributed under the Creative Commons Attribution License, which permits unrestricted use, distribution, and reproduction in any medium, provided the original work is properly cited.

\begin{abstract}
Aim. The purpose of this research is to identify the mechanisms of Shen-Qi compound formula (SQC), a traditional Chinese medicine (TCM), for treating diabetes mellitus (DM) using system pharmacology. Methods. The active components and therapeutic targets were identified, and these targets were analyzed using gene ontology (GO) enrichment analysis, Kyoto Encyclopedia of Genes and Genomes (KEGG) enrichment analysis, and protein-protein interaction (PPI) analysis. Finally, an integrated pathway was constructed to show the mechanisms of SQC. Results. A total of 282 active components and 195 targets were identified through a database search. The component-target network was constructed, and the key components were screened out according to their degree. Through the GO, PPI, and KEGG analyses, the mechanism network of SQC treating DM was constructed. Conclusions. This study shows that the mechanisms of SQC treating DM are related to various pathways and targets. This study provides a good foundation and basis for further in-depth verification and clinical application.
\end{abstract}

\section{Introduction}

Diabetes mellitus (DM) is an endocrine and metabolic disease characterized by persistent hyperglycemia. Its main clinical manifestations include polydipsia, polyphagia, polyuria, and weight change [1]. Due to lifestyle changes, the incidence of DM in China has increased dramatically over the past 30 years. According to epidemiological surveys, China has the largest number of diabetic patients in the world $[2,3]$. There are many chronic complications in the later stage of $\mathrm{DM}$, including macrovascular disease, microvascular disease, and diabetic neuropathy [4]. DM and its associated complications have a serious impact on the health of patients and result in a significant economic and health burden on society.

Although the treatment of DM has long been researched, the management of DM and its chronic complications are still big challenges in clinical practice [5-9]. At present, the main objective is to control blood glucose. Therapeutic agents are mainly composed of insulin and oral hypoglycemic medicines. However, these therapeutic drugs have their drawbacks. Insulin is the most effective hypoglycemic agent, but it increases the risk of hypoglycemia, which in turn increases the risk of cardiovascular disease and other complications $[10,11]$. However, oral hypoglycemic agents cannot achieve the desired state in many cases, which may be due to their adverse reactions or clinical inertia. Therefore, new means of treatment are needed.

In China, the application of traditional Chinese herbal medicine for DM has a long history. Shen-Qi compound formula (SQC) is a traditional Chinese medicine (TCM) formula that is widely used in treating DM in China. It consists of eight herbal medicines: Radix et Rhizoma Ginseng (RRG, rén shèn), Radix Astragali Praeparata cum Melle (RAPM, huáng qí), Radix et Rhizoma Salviae Miltiorrhizae (RRSM, dān shēn), Radix et Rhizoma Rhei (RRR, dà huáng), 
Rhizoma Dioscoreae (RD, huái shān yào), Radix Trichosanthis (RT, tiān hūa fẽ $n$ ), Radix Rehmanniae (RR, di huáng), and Fructus Corni (FC, shān zhū yú). The composition of SC is shown in Table 1. In a clinical trial involving 219 patients with Type 2 DM, SQC treatment over 12 weeks can significantly reduce fasting and postprandial blood glucose levels as well as significantly improve $\beta$ cell function and insulin resistance [12]. Another clinical trial confirmed the effect of SQC on lowering the blood glucose [13]. Clinical research on the treatment of DM complications with SQC is ongoing (ChiCTR1900026372). Regarding the mechanism, in the above clinical studies, SQC was seen to be able to improve outcomes such as superoxide dismutase (SOD), nitric oxide (NO), reactive oxygen species (ROS), malondialdehyde (MDA), total antioxidant capacity (T-AOC), and glucose like peptide-1. Animal experiments showed that the mechanisms might be related to inhibiting adiponectin, reducing the inflammation and other mechanisms $[14,15]$. However, due to the complexity of its components, its comprehensive mechanisms have not yet been fully elucidated.

Network pharmacology comprehensively analyzes many complex networks through a high-throughput analysis and computer calculation. This research idea coincides with the characteristics of the simultaneous effects of multicomponents of TCM. Therefore, it has become an effective tool for the research of traditional Chinese herbal medicine [16].

In this study, we systematically analyzed SQC utilizing network pharmacology to fully elucidate its possible mechanism on DM. Firstly, we screened out the active ingredients of these herbal medicines according to specific criteria. Secondly, the therapeutic targets were obtained. Then, these targets were systematically analyzed, and finally, an integrated pathway was constructed.

\section{Materials and Methods}

2.1. Search and Screening of Chemical Constituents. The workflow for this network pharmacology study can be seen in Figure 1. The screening of the active ingredients of the herbal medicines in SC was conducted on the platform of the TCM System Pharmacology database (TCMSP) (http://lsp. nwu.edu.cn/tcmsp.php) and SymMap (https://www. symmap.org/). TCMSP is a database that is widely used in the network pharmacology research of TCM. It contains information on 499 herbal medicines, 29384 ingredients, and 837 related diseases [17]. This database provides pharmacokinetic information for each compound, which is particularly helpful for us in further screening. SymMap is a database that aims to study the mechanism of TCM from the view point of modern medicine and includes a large amount of information on Chinese herbal medicine, symptoms of TCM, ingredients of medicines, targets, and diseases. In the aspect of drug ingredients, it integrates the information of three databases, TCMID, TCMSP, and TCM-ID, including as many as 19595 ingredients. In terms of disease targets, this database includes information on several commonly used databases, such as HIT, TCMSP, HPO, DrugBank, and NCBI. This database is a useful tool to study the ingredients
TABLE 1: The composition of SC.

\begin{tabular}{|c|c|c|}
\hline Pharmaceutical & Pin yīn & Composition (\%) \\
\hline Radix et Rhizoma Ginseng & rén shēn & 17.4 \\
\hline $\begin{array}{l}\text { Radix Astragali Praeparata cum } \\
\text { Melle }\end{array}$ & huáng qí & 17.4 \\
\hline $\begin{array}{l}\text { Radix et Rhizoma Salviae } \\
\text { Miltiorrhizae }\end{array}$ & dān shēn & 11.6 \\
\hline Radix et Rhizoma Rhei & dà huáng & 2 \\
\hline Rhizoma Dioscoreae & $\begin{array}{l}\text { huái shān } \\
\text { yào }\end{array}$ & 11.6 \\
\hline Radix Trichosanthis & $\begin{array}{l}\text { tiān huā } \\
\quad \text { fẽen }\end{array}$ & 11.6 \\
\hline Radix Rehmanniae Recens & di huáng & 11.6 \\
\hline Fructus Corni & shān $z h \bar{u} y u ́$ & 6.9 \\
\hline
\end{tabular}

and targets of TCM [18]. To further screen the active ingredients in the compound, the results retrieved from the databases were screened by two criteria: oral bioavailability (OB) and drug-likeness (DL).

2.2. Evaluation of $O B$. $O B$ represents the proportion of drugs that enter the human blood circulation after oral administration [19]. OB plays an important role in drug delivery. High OB is usually an important characteristic of a chemical component that can be used as a therapeutic drug [20]. OB $\geq 30 \%$ was selected as the screening criteria, which is recommended by the TCMSP database [21].

2.3. Evaluation of DL. DL is a parameter used to evaluate how "drug-like" a prospective compound is [22]. This concept can help to optimize pharmacokinetics and pharmaceutical properties such as solubility and chemical stability in the drug design [23]. DL was calculated as follows:

$$
\mathbf{T}(\mathbf{A}, \mathbf{B})=\frac{\mathbf{A} \cdot \mathbf{B}}{\|\mathbf{A}\|^{2}+\|\mathbf{B}\|^{2}-\mathbf{A} \cdot \mathbf{B}}
$$

where $\mathbf{A}$ and $\mathbf{B}$ represent the molecular property of the herbal ingredients and average molecular property of all the molecules in the DrugBank database (http://www.drugbank. $\mathrm{ca} /$ ), respectively. DL $\geq 0.18$, which was the average DL index of all the compounds in DrugBank, was applied as the screening criteria for the ingredients. To ensure that the results of the study are comprehensive and reliable, some compounds which do not meet the screening criteria but are reported as the main components in the literature have been retained in our research.

2.4. Literature Search. As some components with pharmacological effects may not be included in the database or do not meet the conditions of $\mathrm{OB}$ and DL, these components will be missed. To avoid this, we searched the medicine of SQC in PubMed for pharmacological research. MeSH terms and free text words were used as the search strategy. Each search term was connected with a Boolean operator to other terms. The time limitation was from January 1, 2000, to February 5, 2020. The full search strategy can be seen in supplementary material 1 . 


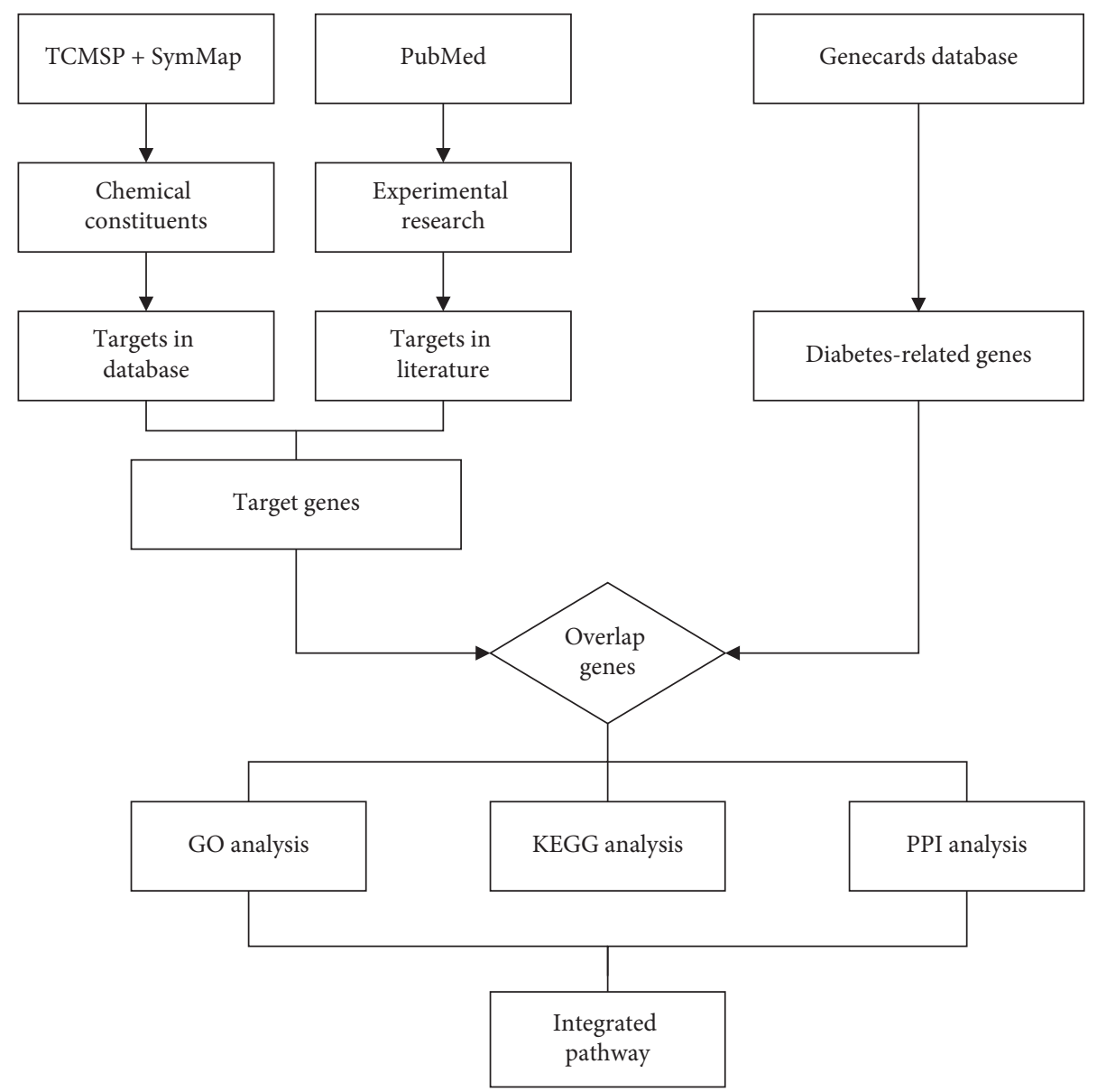

FIGURE 1: Workflow for the present network pharmacology study.

2.5. Screening of Targets for Ingredients. We searched the TCMSP database for protein targets of the chemical constituents we collected. Then, the UniProt Knowledgebase (https:// www.uniprot.org/) was used to search the corresponding human gene names. UniProt Knowledgebase is a protein database with extensive amount of information and resources. It integrates data from three databases: Swiss-Prot, TrEMBL, and PIR-PSD. Its data mainly come from the protein sequence obtained after the completion of the genome sequencing project. It contains a large amount of information about the biological functions of proteins from the literature. We retrieved information about proteins and replaced the protein targets with corresponding gene targets in this database.

2.6. Screening of Targets for DM. We then searched about the targets for DM. These were retrieved from the Human Gene Database (Genecards database, https://www.genecards.org/). GeneCards is a searchable, integrative database that provides comprehensive, user-friendly information on all annotated and predicted human genes. It integrates gene-centric data from about 150 databases, including NCBI, UCSC, and Nsembl [24-26]. Diabetes-related target genes were retrieved using the keywords "diabetes" or "diabetes mellitus". The score ranks diseases by how closely they are associated with the gene, factoring in the relative reliability of the sources, and a criteria score $>5$. This is widely used in network pharmacology research and applied to further screen the target genes $[27,28]$.

2.7. Protein-Protein Interaction Network Construction. The protein-protein interaction (PPI) network analysis helps study the molecular mechanism of diseases and discover new drug targets from a systematic perspective. We constructed a PPI network for the protein targets of SQC, which we retrieved in the TCMSP database. This was done using the string database (https://string-db.org/). This database is used for searching and predicting protein interactions. It can be applied to 2031 species and includes 9.6 million protein interactions and 13.8 million protein interactions. In addition to the experimental data, the results of text mining from PubMed abstracts, and the synthesis of other database data, it also uses bioinformatic methods to predict the results. This is one of the most diverse and informative websites for PPI analysis. The line thickness indicates the strength of data support, that is, confidence. The minimum required interaction score is medium confidence (0.400), which is also the default threshold.

2.8. Gene Ontology Enrichment and Kyoto Encyclopedia of Gene and Genome Pathway Enrichment. To elucidate the mechanism of SQC in treating DM, we collected the 
intersected target genes for gene ontology (GO) enrichment and Kyoto Encyclopedia of Gene and Genome (KEGG) pathway enrichment analysis. GO enrichment is the description of genes in different dimensions and levels such as the cellular components (CC), biological process (BP), and molecular function (MF). Through the GO analysis, we can get a better understanding of genes [29]. KEGG analysis refers to the analysis of the pathways involved in genes. Through the KEGG analysis, we can fully understand the relevant pathways in biological processes. GO enrichment and KEGG pathway enrichment analyses were performed on the Database for Annotation, Visualization, and Integrated Discovery (DAVID 6.8, https://david.ncifcrf.gov/). DAVID is a robust, publicly available database that aims to illuminate how environmental chemicals affect human health [30,31]. In the results of the GO and KEGG analyses, the $P$ value indicates whether the pathway is significantly related to SQC, and the count value reflects the number of targets on the pathway. The larger the count value is, the more targets SQC has on the pathway. We filtered according to a $P$ value of $<0.01$ and arranged the filtered results according to the count. For the GO analysis, we selected the top five results for each item to display. For the KEGG analysis, to provide readers with a more comprehensive understanding of the mechanism of SQC, the top 25 entries in the KEGG pathways were exhibited.

2.9. Network Construction. To display the above results more vividly, compound-target (C-T) network and target-pathway (T-P) network diagrams for the above results were constructed. These network diagrams were drawn using Cytoscape version 3.6.1, which can graphically display the network and edit it [32]. In these graphs, compounds and targets are represented by nodes and their interactions are reflected by edges.

2.10. Signal Pathway Construction of SQC in Treating DM. We excluded the pathways that were unrelated to diabetes among the top 25 pathways. The rest of the pathways were considered to be the main mechanisms. Then, we looked up the KEGG database to understand the specific processes of these mechanisms. Due to the complexity of mechanisms, only the most commonly used and representative molecules in pathways and proven interactions were selected in the construction of the signal pathway. Special attention was paid to the interaction between signal pathways. In this way, we integrated the rest of the diabetic signal pathways and constructed the pathway network of SQC in the treatment of DM.

\section{Results}

3.1. Active Ingredients in SQC. After a preliminary search, a total of 959 chemical ingredients were found in the TCMSP database and 1183 ingredients in SymMap. The information of ingredients retrieved in TCMSP and SymMap can be viewed in the supplementary materials 2 and 3, respectively. Then, the retrieval results of the two databases are combined.
A total of 516 ingredients remained including 70 ingredients in rén shēn, 19 in dì huáng, 103 in shān zhü yú, 175 in dān shēn, 52 in huáng qí, 9 in tiān huā fẽn, 154 in huái shān yào, and 19 in dahuang. After a literature search, 30 additional ingredients were added to our study including 10 in RRG (Ginsenoside Re [33], ginseng total saponin [34], Ginsenoside- $\operatorname{Rg} 3$ [35-37], ginsenoside $\operatorname{Rh} 2$ [38, 39], ginsenoside Rb2 [40], Ginsenoside Rb1 [41-43], Compound K [44], Ginsenoside Rg1 [45, 46], Ginseng polysaccharides [47, 48], and Malonylginsenoside Rc), 3 in RAPM (Astragalus polysaccharide [49-66], Astragaloside Iv [67-72], and astragalin [73]), 4 in RRSM (danshensu [74], Lithospermic acid B [75, 76], Magnesium Lithospermate B [77, 78], and salvianolic acid B [79-85]), 3 in RRR (rhein [86], emodin [87, 88], and Argirein [89]), 2 in RD (Allantoin [90, 91], Dioscorea opposita Polysaccharide [92-94]), 1 in RR (catalpol [95-103]), 7 in FC (5-hydroxymethylfurfural [104], Iridoid glycoside [105], Loganin [106], 7-O-Galloyl-Dsedoheptulose [107, 108], total triterpene acids [109], iridoid total glycoside [110], and cornuside). After removing the duplicates, a total of 352 nonrepeating ingredients were retrieved.

3.2. Target Retrieval and Analysis. Based on the above results, we further searched the targets of these ingredients. A total of 5201 targets were retrieved. Four-hundred and fortyeight targets remained after eliminating the duplicate targets. Then, DM-related targets were searched in the platform, Genecards. A total of 1244 targets were retrieved in the database using the keyword "diabetes". By intersecting the target of SQC with that of DM, a total of 195 overlapping target genes were screened out. The targets of SQC and DM can be viewed in supplementary material 4 . These targets are considered to be the active targets of SQC in the treatment of $\mathrm{DM}$, as shown in Figure 2(a). Then, we further compared the active target we obtained with the original targets of SQC. Components that do not have active targets will be considered as ineffective ingredients. Eventually, 282 ingredients were retained, which were considered to be active ingredients in the treatment of DM by SQC. These results could help us understand the mechanisms likely to be involved in its therapeutic effect. A network of active compound-target was constructed according to these results (Figure 2(b)). Then, we ranked these ingredients according to the degree. The top 10 ingredients included kaempferol, supervisolin, Gly, beta-sitosterol, stigmasterol, astragalus polysaccharide, oleic acid, isorhamnetin, and aloe-emodin. Figure 2(c) shows the interaction between these ingredients and their targets.

3.3. GO Enrichment and KEGGAnalysis. To further elucidate the molecular mechanism(s) of SQC in the treatment of DM, GO enrichment and KEGG pathway analyses were performed on the selected candidate targets in the CTD database. The GO analysis describes the function of candidate targets from three aspects: biological process (BP), cellular component (CC), and molecular function (MF). A $P$ value of $<0.01$ was considered statistically significant. The complete 


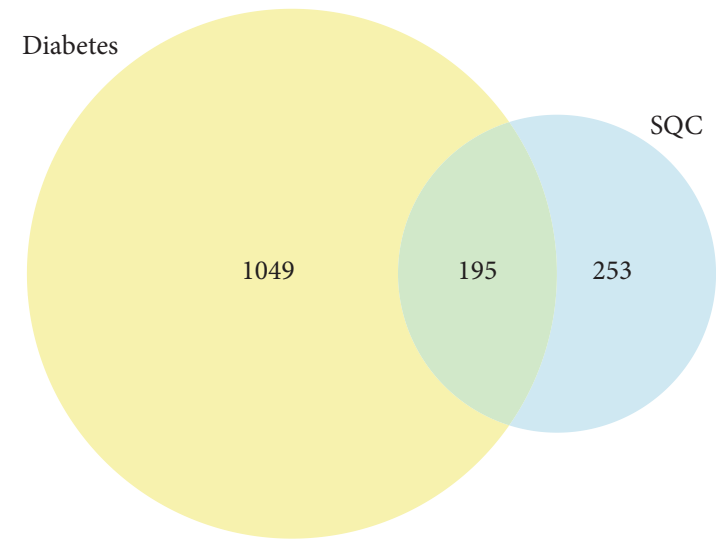

(a)

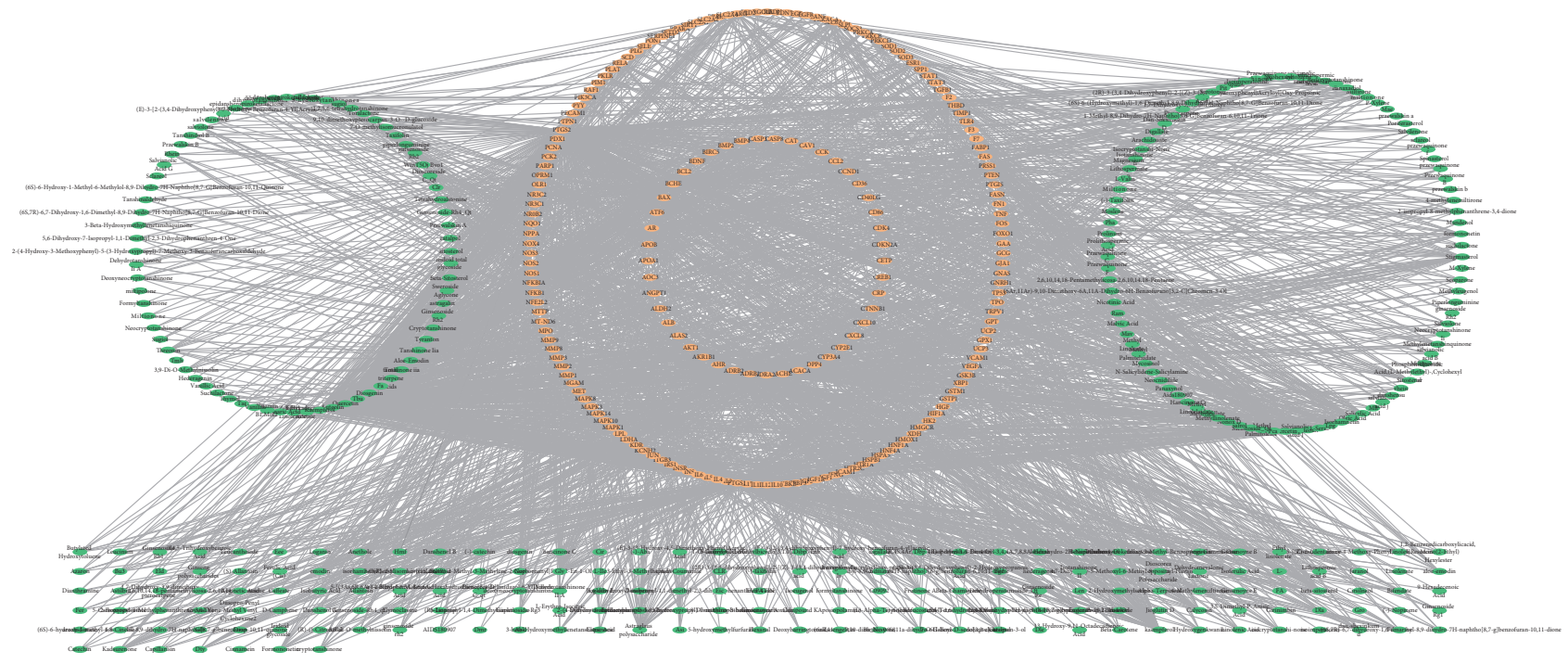

(b)

Figure 2: Continued. 


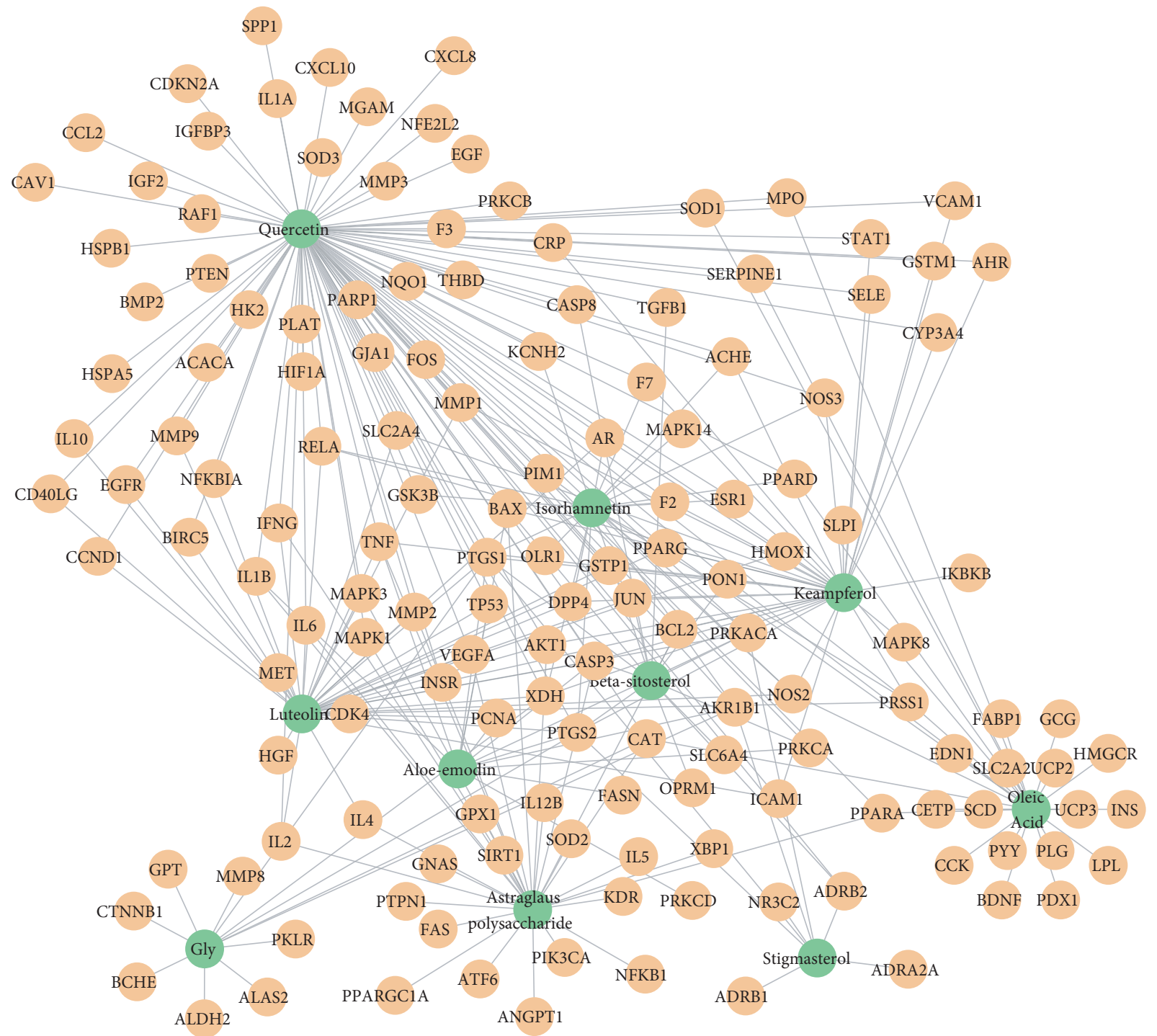

(c)

FIgURE 2: Network construction. (a) Venn diagram of diabetes-related targets and SQC-related targets. The yellow circle represents diabetes-related targets, and the blue circle represents SQC-related targets. (b) The network of compound-target in SQC. The green nodes represent the active compound in SQC. The blue nodes represent the potential targets. The edges represent the interaction between the blue and green nodes. (c) Top 10 ingredients and their targets. The green nodes represent the ingredients, the orange nodes represent the targets, and the edges represent the interaction between them.

results of the GO enrichment analysis are displayed in supplementary materials 5 . The top five results for the three aspects are shown in Figure 3. According to the results of the GO enrichment analysis, the biological processes involved are mainly the positive regulation of transcription from RNA polymerase II promoter (GO: 0045944), negative regulation of apoptotic process (GO: 0043066), transcription, DNAtemplated (GO: 0006351), inflammatory response (GO: 0006954), positive regulation of transcription, and DNAtemplated (GO: 0045893). The enriched molecular functional ontologies mainly include transcription factor activity, sequence-specific DNA binding (GO: 0003700), DNA binding (GO: 0003677), sequence-specific DNA binding (GO: 0043565), cytokine activity (GO: 0005125), and growth factor activity (GO: 0008083). The cellular component analysis showed that extracellular space (GO: 0005615), membrane raft (GO: 0045121), extracellular matrix (GO: 0031012), cytosol (GO: 0005829), and external side of the plasma membrane (GO: 0009897) were the most important.

Subsequently, we analyzed the KEGG pathway of the candidate genes. The results of the KEGG analysis are displayed in supplementary material 6 . We sequenced these pathway analysis results by the count of genes. Figure 4 shows the 25 most prominent signal pathways. According to these results, the most important pathways related to treating of diabetes by SQC are PI3K-Akt, HIF-1, TNF, insulin resistance, FoxO, MAPK, AMPK, and insulin signal pathways. 


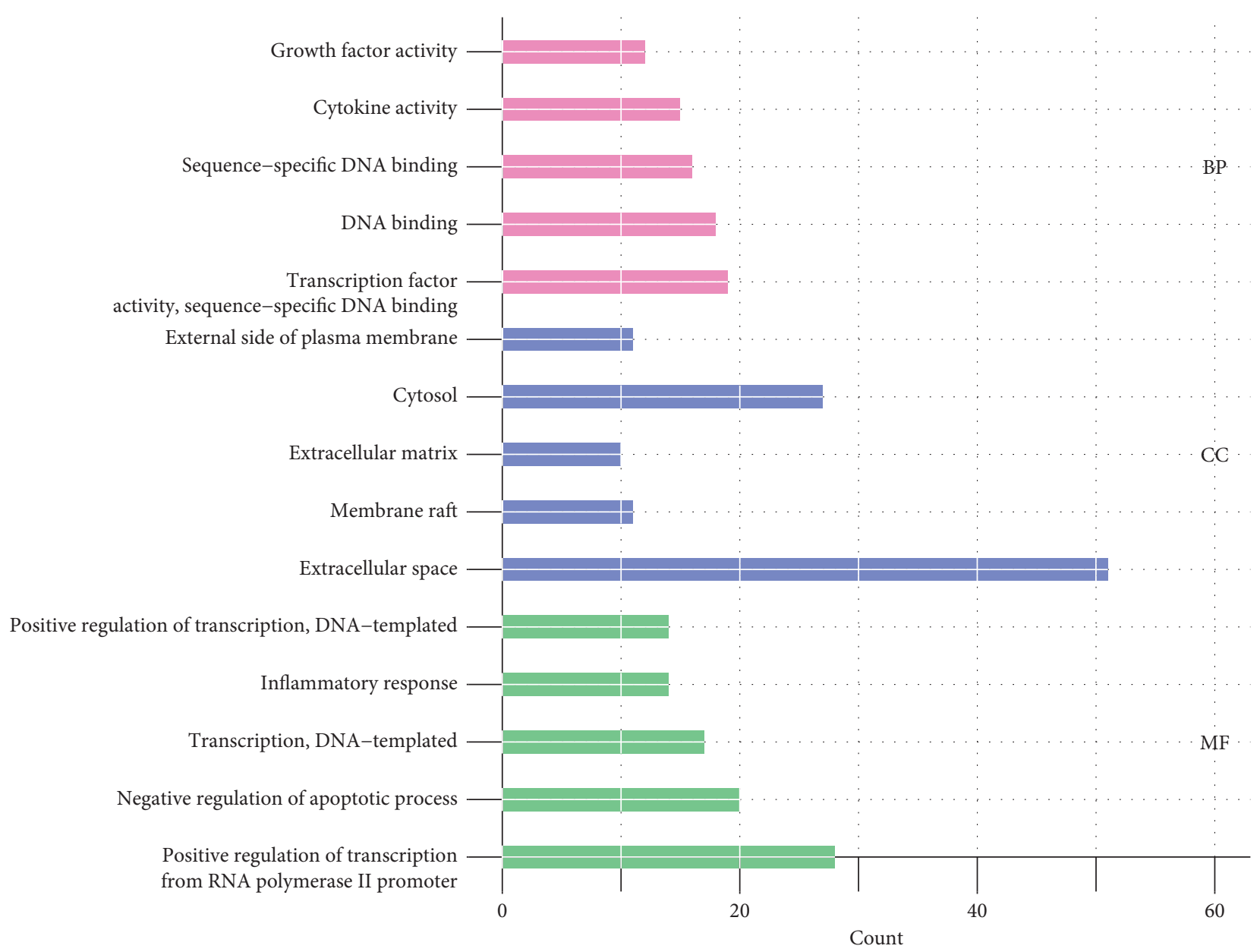

FIGURE 3: GO enrichment analysis of the SQC-related target genes in the treatment of DM. The $X$-axis represents the gene count of each GO term, and the $Y$-axis represents the categories in the cellular component, molecular function, and biological process. All the categories were screened using the criteria of a $P$ value $<0.01$.

To show the relationship between these 25 pathways and their related genes, we have mapped the T-P network (Figure 5). In this, the two outer circles represent the genes associated with pathways. The innermost circle represents the most prominent 25 signal pathways.

3.4. PPI Network for the Targets of SQC. Then, we conducted a PPI network analysis. As is shown in Figure 6, there are 164 nodes, 3684 edges, and an average node of 44.9. The top ten genes according to the degree are as follows: AKT1, interleukin-6 (IL6), albumin (ALB), insulin (INS), vascular endothelial growth factor $\alpha$ (VEGFA), tumor necrosis factor (TNF), tumor protein P53 (TP53), caspase 3 (CASP3), mitogen-activated protein kinase 3 (MAPK3), and mitogen-activated protein kinase 8 (MAPK8). These genes are considered to play an important role in treating of DM (Figure 7).

3.5. Construction of an Integrated Pathway for SQC. According to the KEGG results, an integrated pathway was constructed by combining the key pathways in the treatment of DM, including PI3k-Akt, TNF, HIF- $\alpha$, insulin resistance,
FoxO, toll-like Ras, Rap-1, AMPK, MAPK, and cAMP signal pathways (Figure 8).

\section{Discussion}

In this study, the mechanism of SQC in the treatment of DM was comprehensively identified using network pharmacology. Some interesting results were found in our research, which will be helpful in our further investigations.

A total of 352 nonrepeating ingredients including 282 active ingredients were identified through database and literature search. The components of TCM formula include not only the compounds contained in each herbal medicine but also the new components generated in the preparation of the formula. In this research, new ingredients generated during the preparation process were not investigated. In future research, more attention should be paid to these newly generated compounds.

There were 150 targets in all of the top 10 ingredients, and all of these 10 ingredients have multiple targets. The analysis of these targets shows that these mainly exert antidiabetic effects through various pathways, such as the TNF, PI3K/Akt, insulin resistance, and HF-1 signal pathways. By 


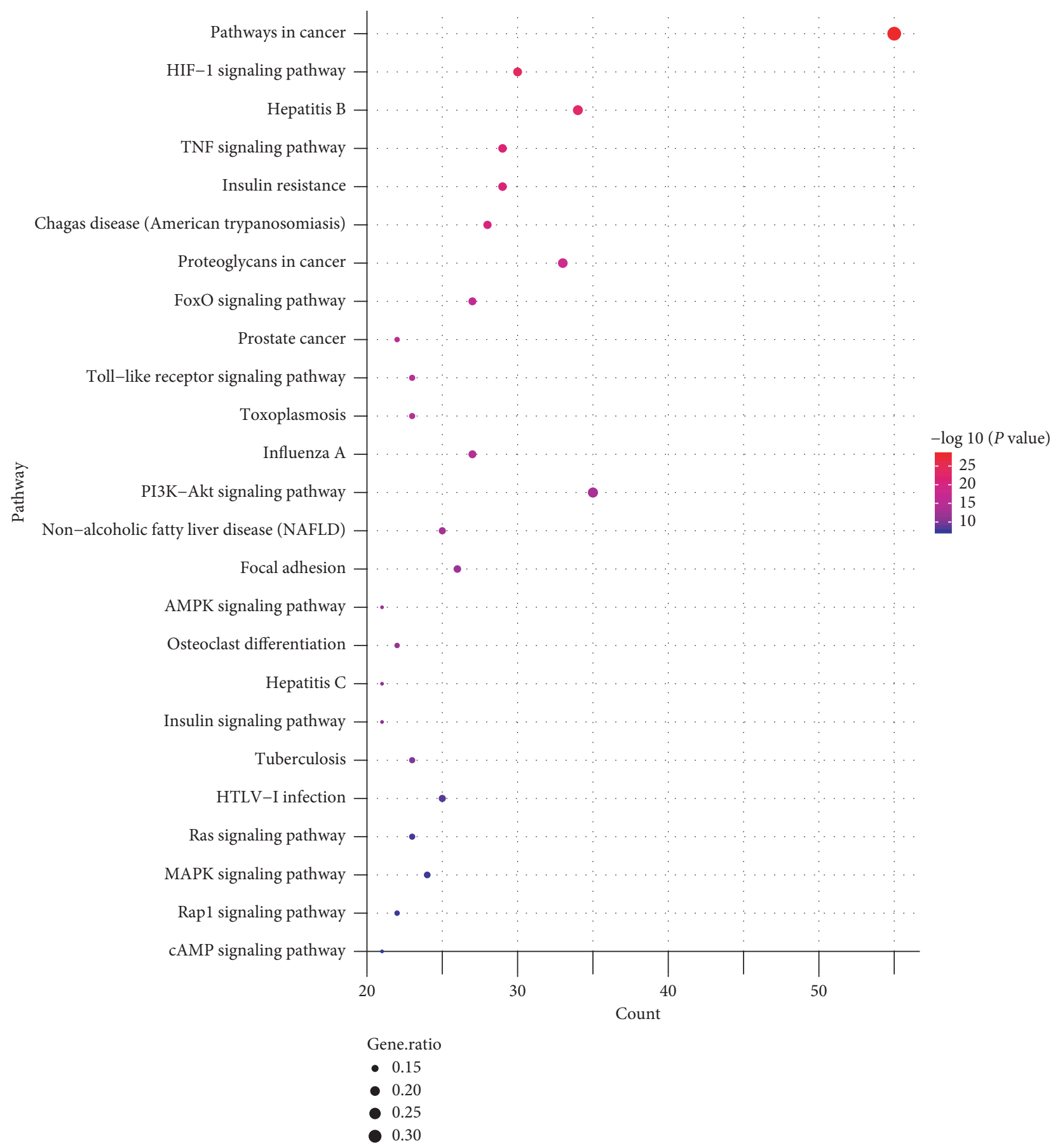

FIGURE 4: Bubble chart of the KEGG enrichment analysis of the SQC-related target genes in the treatment of DM. The $X$-axis represents the count of genes of each GO term, the $Y$-axis represents the categories in the KEGG analysis, the size of the nodes represents the gene ratio, and the color of the nodes was determined by the value of $-\log 10$ ( $P$ value).

directly affecting several key proteins in these pathways, such as Akt, TNF, and MAPK, these ingredients can directly affect the activity of the abovementioned multiple diabetes-related signal pathways. Multiple ingredients act on the same target, indicating that these components have synergistic effects in treating diabetes.

The GO analysis describes the target of SQC from the aspects of the biological process, molecular function, and cell components. In terms of cell components, the related targets of SQC are located in the extracellular space. If the product of a gene is secreted from cells to tissue fluid or blood, then generally, the gene will be annotated as extracellular space. In the physiological process of diabetes, insulin is secreted from the cell after synthesis. Also, diabetes is an inflammatory disease. Many inflammatory cytokines are secreted into the blood from the cell. These targets were classified as extracellular space. The results of the GO analysis suggest that SQC may have a regulatory effect on these processes. 


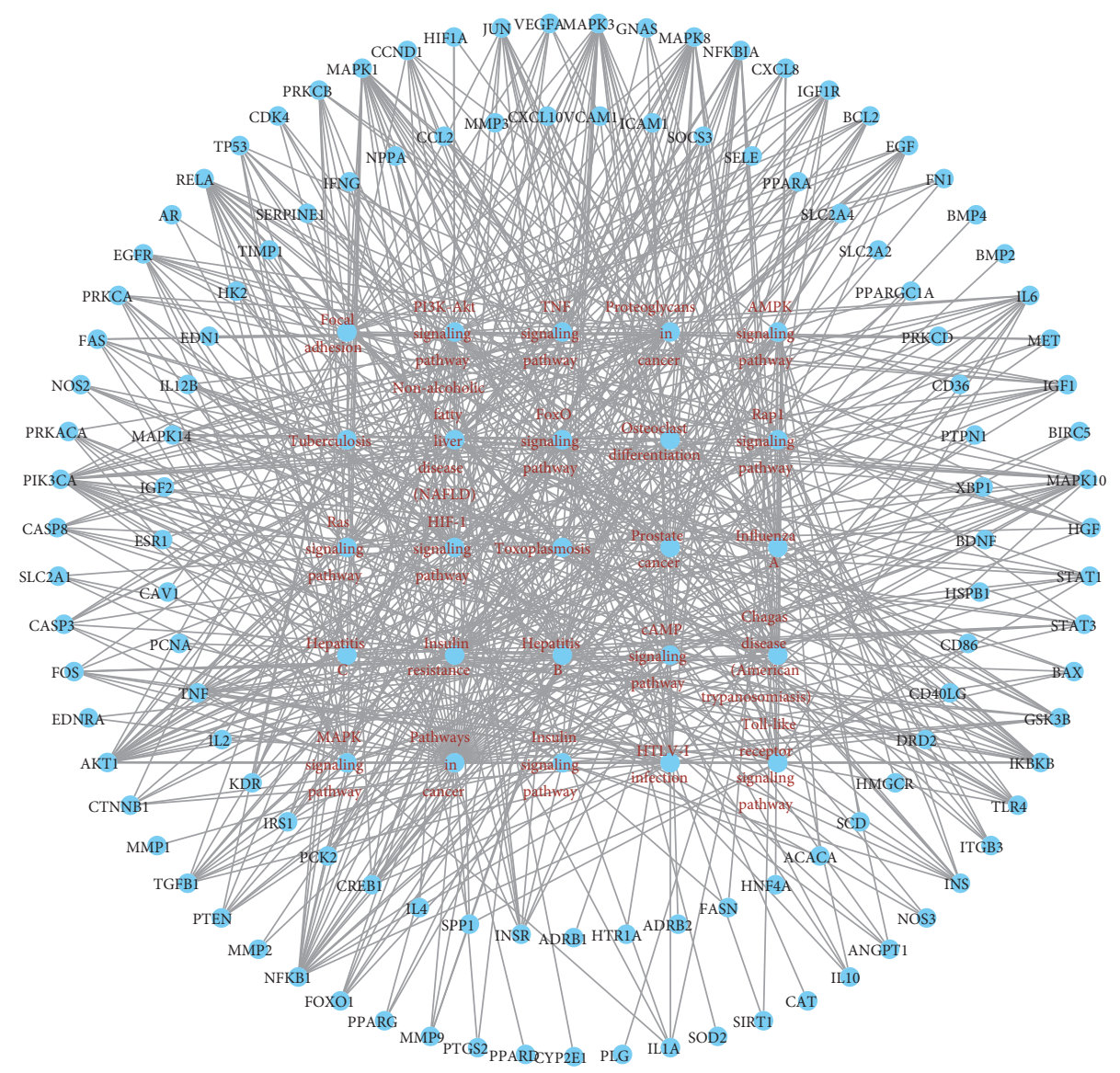

FIGURE 5: The network of the target-pathway in SQC. The red nodes represent the pathway of SQC in the treatment of DM. The blue nodes represent the target genes. The edges represent the interaction between the red and blue nodes.

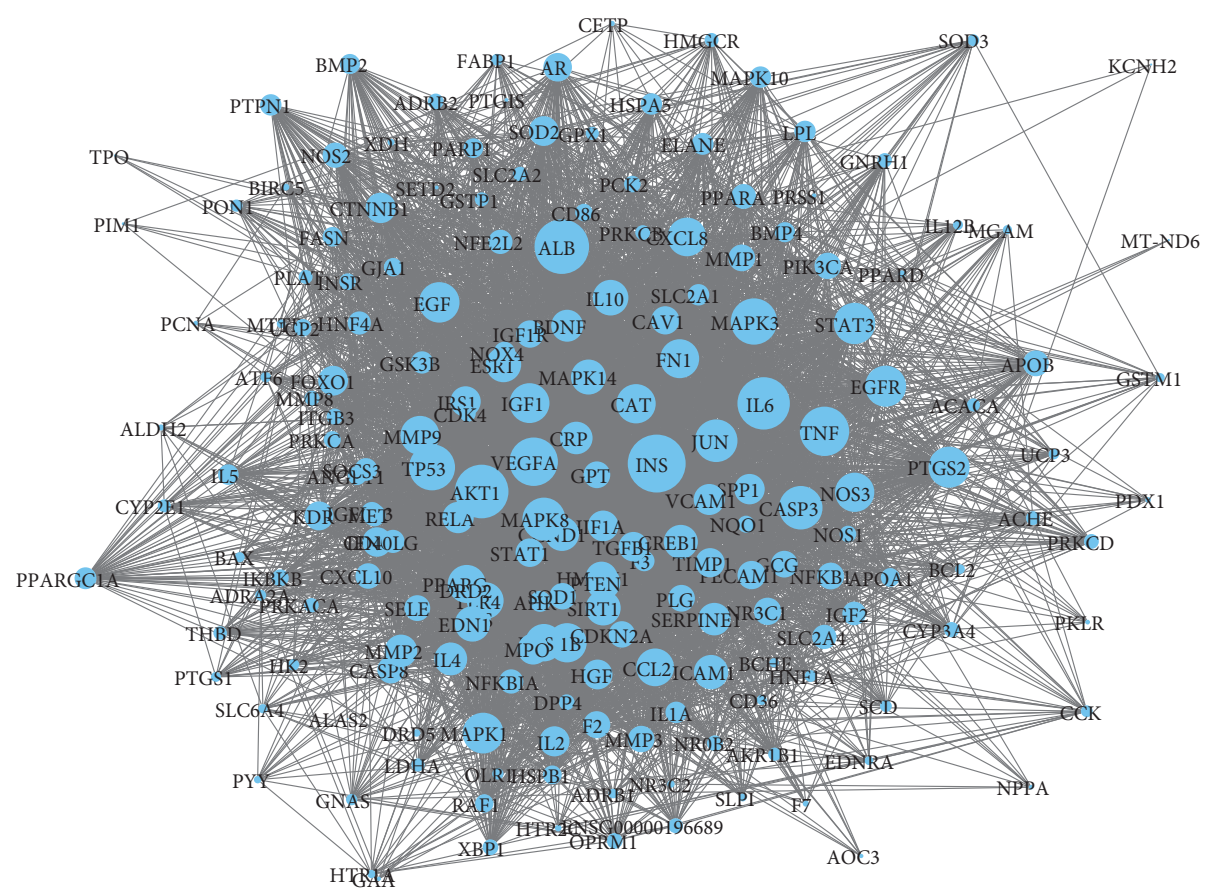

Figure 6: PPI network of the target genes. The size of the nodes is proportional to their degrees. 


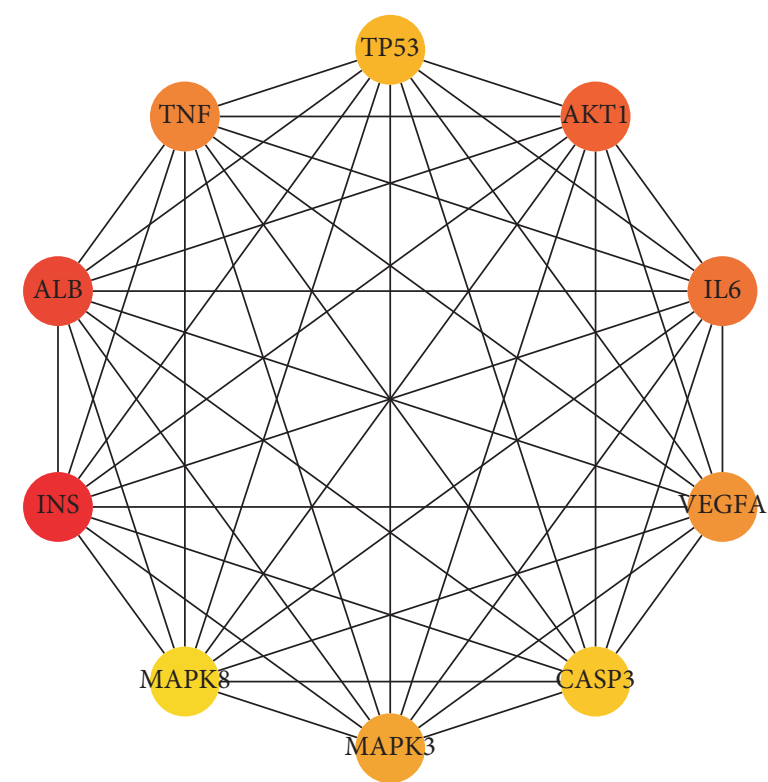

Figure 7: Top 10 hub genes in the PPI network.

By analyzing the molecular function of the target gene, we can further understand the biological process of its main ingredients. The main molecular function of SQC is to regulate RNA polymerase, affect apoptosis, and reduce inflammation. This result is consistent with the biological process. The transcription process of an organism is mainly initiated by the formation of transcription initiation complex by transcription factors and RNA polymerase II, which are the bound to the downstream DNA. In DM, the FoxO transcription factor is a key molecule in the insulin or insulin-like growth factor signal pathway, and the p65 transcription factor is an important transcription factor that helps to regulate inflammation. The PI3K/Akt signal pathway can affect many downstream transcription factors and biological processes, including apoptosis and inflammation. Chronic inflammation and apoptosis of $\beta$ cells will have a significant impact on the insulin signal of cells and eventually lead to diabetes. The results of the GO analysis indicated that SQC might have an impact on biological processes like $\beta$ cell apoptosis chronic inflammation.

The KEGG analysis provided a further understanding of the mechanism of SQC based on the GO analysis. The PI3K/ Akt signal pathway is one of the most important signal pathways in the pathogenesis of diabetes. It is a significant pathway in cell mobilization, migration, differentiation, and antiapoptosis. It also plays an important role in the regulation of glucose transport, glycogen synthesis, glycolysis, and gluconeogenesis, as well as in the process of protein synthesis and fat decomposition. The KEGG analysis showed that SQC has $35 \mathrm{PI} 3 \mathrm{~K} / \mathrm{Akt}$-related targets. This indicates that SQC can regulate the apoptosis and metabolism of diabetes.

Chronic inflammation can affect the insulin sensitivity of the body and even lead to the occurrence of insulin resistance. TNF is one of the most important signal pathways in inflammation. It can not only activate the downstream inflammatory response, leading to the aggravation and chronicity of inflammation but also directly affect the insulin signal pathway and aggravate insulin resistance. Furthermore, the TNF pathway can directly affect the MAPK signal pathway, which is closely related to cell proliferation and apoptosis. Through these mechanisms, the activity of the TNF signal pathway has a significant impact on DM. SQC can improve the insulin resistance of DM by affecting the TNF signal pathway, which is consistent with the previous clinical research results.

Hypoxia-inducible factor-1 $\alpha$ (HIF-1 $\alpha$ ) is an important transcription factor, which can regulate the expression of many downstream target genes and participate in hypoxia adaptation, angiogenesis, immune response, apoptosis, and other reactions. It plays an important role in diabetes. The changes of HIF-1 $\alpha$ expression level in $\mathrm{DM}$, together with the structural and functional abnormalities caused by the covalent modification of glyoxal, ultimately lead to disorder of the signal pathway regulation, such as angiogenesis and apoptosis. These are important molecular events in the occurrence and progress of diabetes and its complications. It was found that the TNF, PI3K/Akt, and MAPK signal pathways can interact with the HIF signal pathway, leading to the gradual progress of the disease.

Among the signal pathways we obtained through the KEGG analysis, the toll-like receptor and cAMP and Ras pathways are important signal pathways for organisms, which can participate in a variety of biological processes and have mutual relationships with multiple signal pathways. For example, toll-like receptor-mediated pattern recognition can rapidly activate the immune response, $\mathrm{NF}-\kappa \mathrm{B}$ signal pathway, and MAPK signal pathway downstream of TNF, thus leading to inflammation and apoptosis.

The PPI analysis further explored the interaction of these targets. From these results, we can know which targets play a more important role. TNF, Akt, MAPK, and other targets 


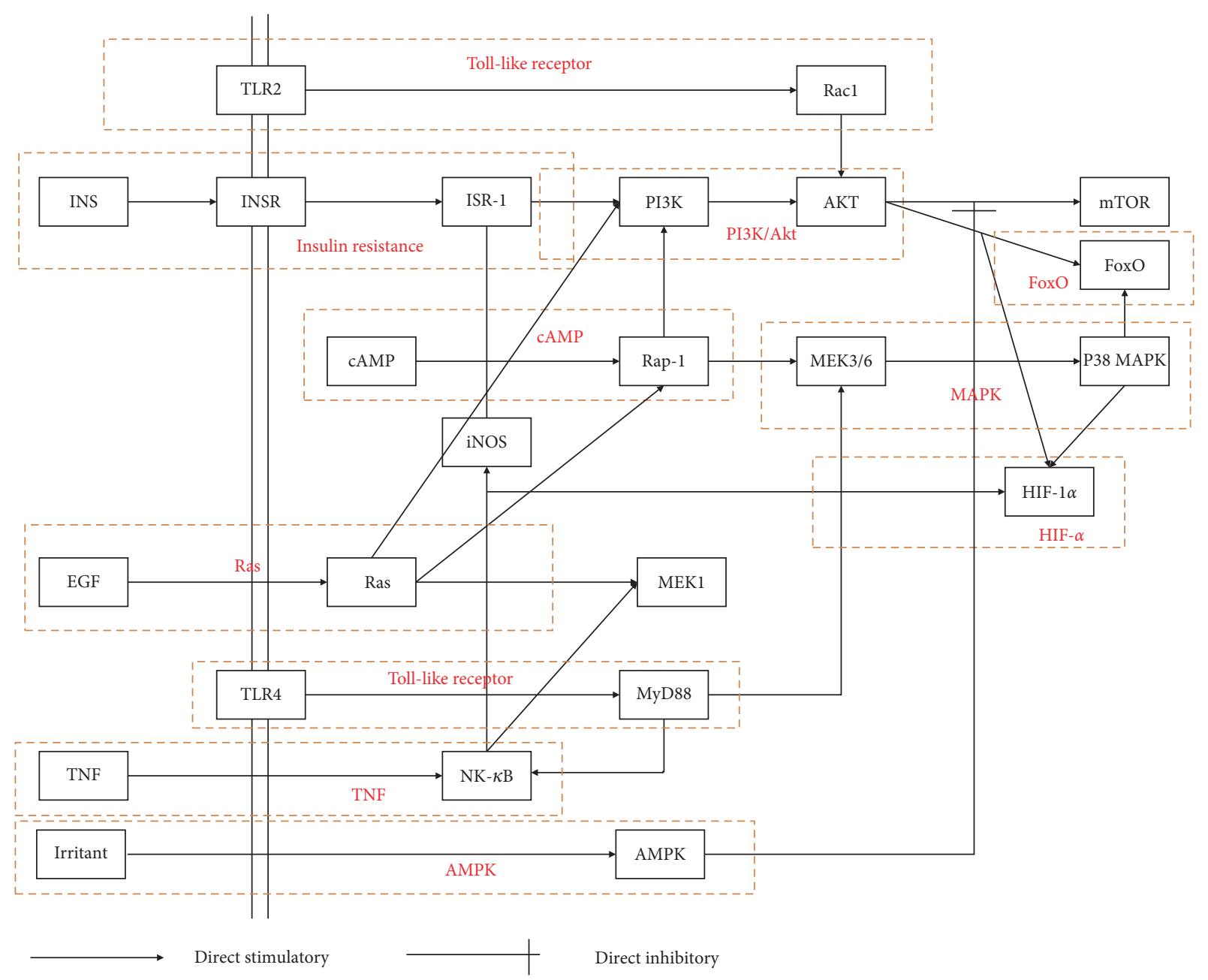

FIGURE 8: The integrated pathway and potential mechanism(s) of SQC in the treatment of DM.

are the most important proteins. This result is consistent with the results of the KEGG analysis.

\section{Conclusions}

This research systematically investigated the ingredients and targets of SQC in the treatment of DM through the combination of network pharmacology and a literature search. A total of 282 components and 195 targets were obtained. The multitarget mechanisms of SQC were studied using the GO, KEGG, and PPI analyses. This study provides a solid foundation for further experimental verification and clinical application. Further experimental verification is needed to confirm the result of this current study.

\section{Data Availability}

The complete information of active ingredients, MOL_ID, molecule names, targets, and gene code, and GO and KEGG analysis results can be seen in supplementary materials.

\section{Disclosure}

Zhipeng $\mathrm{Hu}$ and Maoyi Yang are regarded as co-first authors.

\section{Conflicts of Interest}

The authors declare that there are no conflicts of interest regarding the publication of this paper.

\section{Authors' Contributions}

Zhipeng $\mathrm{Hu}$ and Maoyi Yang contributed equally to this work.

\section{Acknowledgments}

This work was supported by the National Natural Science Foundation of China (Nos. 81774302 and 81703953) and Science and Technology Program of Sichuan Province (No. 2019YFS0022). In addition, we would like to thank Editage (http://www.editage.cn) for English language editing. 


\section{Supplementary Materials}

Supplementary material 1: the search strategies for eight herbal medicines in SQC. Supplementary material 2: the information of ingredients retrieved in TCMSP. Supplementary material 3: the information of ingredients retrieved in SymMap. Supplementary material 4: summarized target information, including the targets of SQC and diabetes. Supplementary material 5: raw data of GO enrichment analysis. Supplementary material 6: raw data of KEGG enrichment analysis. (Supplementary Materials)

\section{References}

[1] N. A. J. Krentz and A. L. Gloyn, "Insights into pancreatic islet cell dysfunction from type 2 diabetes mellitus genetics," Nature Reviews Endocrinology, vol. 16, no. 4, pp. 202-212, 2020.

[2] N. H. Cho, J. E. Shaw, S. Karuranga et al., "IDF Diabetes Atlas: global estimates of diabetes prevalence for 2017 and projections for 2045," Diabetes Research and Clinical Practice, vol. 138, pp. 271-281, 2018.

[3] C. Hu and W. Jia, "Diabetes in China: epidemiology and genetic risk factors and their clinical utility in personalized medication," Diabetes, vol. 67, no. 1, pp. 3-11, 2018.

[4] J. A. Beckman and M. A. Creager, "Vascular complications of diabetes," Circulation Research, vol. 118, no. 11, pp. 1771-1785, 2016.

[5] D. M. Nathan, P. H. Bennett, J. P. Crandall et al., "Does diabetes prevention translate into reduced long-term vascular complications of diabetes?" Diabetologia, vol. 62, no. 8, pp. 1319-1328, 2019.

[6] E. Quattrocchi, T. Goldberg, and N. Marzella, "Management of type 2 diabetes: consensus of diabetes organizations," Drugs in Context, vol. 9, p. 212607, 2020.

[7] F. Herrera-Gómez, M. Asensio-González, A. GonzálezLópez, and F. J. Álvarez, "Effects of intensive control of glycemia on clinical kidney outcomes in type 2 diabetes patients compared with standard control: a meta-analysis," Frontiers in Pharmacology, vol. 8, p. 845, 2017.

[8] J. D. Newman, A. Z. Schwartzbard, H. S. Weintraub, I. J. Goldberg, and J. S. Berger, "Primary prevention of cardiovascular disease in diabetes mellitus," Journal of the American College of Cardiology, vol. 70, no. 7, pp. 883-893, 2017.

[9] N. Sattar, J. J. McMurray, and A. Y. Cheng, "Cardiorenal risk reduction guidance in diabetes: can we reach consensus?" The Lancet Diabetes \& Endocrinology, vol. 8, no. 5, pp. 357-360, 2020.

[10] K. A. Connelly, A. T. Yan, L. A. Leiter, D. L. Bhatt, and S. Verma, "Cardiovascular implications of hypoglycemia in diabetes mellitus," Circulation, vol. 132, no. 24, pp. 23452350, 2015.

[11] A. Goto, M. Goto, T. Yasuo et al., "Association between severe hypoglycemia and cardiovascular disease risk in Japanese patients with type 2 diabetes," Journal of the American Heart Association, vol. 5, Article ID e002875, 2016.

[12] X. Zhang, Y. Liu, D. Xiong, and C. Xie, "Insulin combined with Chinese medicine improves glycemic outcome through multiple pathways in patients with type 2 diabetes mellitus," Journal of Diabetes Investigation, vol. 6, no. 6, pp. 708-715, 2015.
[13] Y. Zhang, H. Y. Xie, Y. Tian, L. Y. Zhou, R. Yan, and C. G. Xie, "Efficacy of Shenqi compoundparticle on blood glucose and oxidative stress compared with metformin for patients with newly siagnosed type 2 diabetes mellitus: randomized clinical trial," International Journal of Clinical and Experimental Medicine, vol. 12, no. 7, pp. 8271-8280, 2019.

[14] Y. Liu, J. Kang, H. Gao et al., "Exploration of the effect and mechanism of ShenQi compound in a spontaneous diabetic rat model," Endocrine, Metabolic \& Immune Disorders-Drug Targets, vol. 19, no. 5, pp. 622-631, 2019.

[15] H. Gao, Y. Duan, X. Fu et al., "Comparison of efficacy of SHENQI compound and rosiglitazone in the treatment of diabetic vasculopathy analyzing multi-factor mediated disease-causing modules," PLoS One, vol. 13, Article ID e0207683, 2018.

[16] S. Tian, Y. Li, D. Li et al., "Modeling compound-target interaction network of traditional Chinese medicines for type II diabetes mellitus: insight for polypharmacology and drug design," Journal of Chemical Information and Modeling, vol. 53, no. 7, pp. 1787-1803, 2013.

[17] J. Ru, Li Peng, J. Wang et al., "TCMSP: a database of systems pharmacology for drug discovery from herbal medicines," Journal of Cheminformatics, vol. 6, p. 13, 2014.

[18] Y. Wu, F. Zhang, K. Yang et al., "SymMap: an integrative database of traditional Chinese medicine enhanced by symptom mapping," Nucleic Acids Research, vol. 47, 2019.

[19] H. Liu, J. Wang, W. Zhou, Y. Wang, and L. Yang, "Systems approaches and polypharmacology for drug discovery from herbal medicines: an example using licorice," Journal of Ethnopharmacology, vol. 146, no. 3, pp. 773-793, 2013.

[20] M. A. Alam, I. Al-Jenoobi Fahad, M. Al-Mohizea Abdullah et al., "Understanding and managing oral bioavailability: physiological concepts and patents," Recent Patents on AntiCancer Drug Discovery, vol. 10, pp. 87-96, 2015.

[21] X. Xu, W. Zhang, C. Huang et al., "A novel chemometric method for the prediction of human oral bioavailability," International Journal of Molecular Sciences, vol. 13, no. 6, pp. 6964-6982, 2012.

[22] W. P. Walters and M. A. Murcko, "Prediction of "druglikeness"” Advanced Drug Delivery Reviews, vol. 54, no. 3, pp. 255-271, 2002.

[23] S. Tian, J. Wang, Y. Li, D. Li, L. Xu, and T. Hou, "The application of in silico drug-likeness predictions in pharmaceutical research," Advanced Drug Delivery Reviews, vol. 86, pp. 2-10, 2015.

[24] M. Rebhan, V. Chalifa-Caspi, J. Prilusky et al., "GeneCards: integrating information about genes, proteins and diseases," Trends in Genetics, vol. 13, no. 4, p. 163, 1997.

[25] S. Marilyn, D. Irina, J. Alexander et al., "GeneCards version 3: the human gene integrator," Database, vol. 2010, p. baq020, 2010.

[26] F. Simon, S. Zimmerman, A. Kohn et al., "Genic insights from integrated human proteomics in GeneCards," Database, vol. 2016, 2016.

[27] G. Stelzer, R. Rosen, I. Plaschkes et al., "The GeneCards suite: from gene data mining to disease genome sequence analysis," Current Protocols in Bioinformatics, vol. 54, pp. 1.30.11.30.33, 2016.

[28] L. Yang, W. Liu, Z. Hu et al., "A systems pharmacology approach for identifying the multiple mechanisms of action of the wei pi xiao decoction for the treatment of gastric precancerous lesions," Evidence-Based Complementary and Alternative Medicine, vol. 2019, p. 15, 2019. 
[29] M. Ashburner, C. A. Ball, J. A. Blake et al., "Gene Ontology: tool for the unification of biology," Nature Genetics, vol. 25, no. 1, pp. 25-29, 2000.

[30] D. W. Huang, B. T. Sherman, and R. A. Lempicki, "Systematic and integrative analysis of large gene lists using DAVID Bioinformatics Resources," Nature Protocols, vol. 4, no. 1, pp. 44-57, 2009.

[31] D. W. Huang, B. T. Sherman, X. Zheng et al., "Extracting biological meaning from large gene lists with DAVID," Current Protocols in Bioinformatics, vol. 27, no. 1, pp. 1-13, 2009.

[32] G. Su, H. Morris John, D. Barry et al., "Biological network exploration with Cytoscape 3," Current Protocols in Bioinformatics, vol. 47, 2014.

[33] W. C. S. Cho, T.-T. Yip, W.-S. Chung et al., "Altered expression of serum protein in ginsenoside Re-treated diabetic rats detected by SELDI-TOF MS," Journal of Ethnopharmacology, vol. 108, no. 2, pp. 272-279, 2006.

[34] T.-S. Ha and D.-S. Ha, "Ginseng total saponin attenuates podocyte apoptosis induced by diabetic conditions through the recovery of CD2-associated protein," Journal of Medicinal Food, vol. 22, no. 2, pp. 170-177, 2019.

[35] K. S. Kang, N. Yamabe, H. Y. Kim, J. H. Park, and T. Yokozawa, "Effects of heat-processed ginseng and its active component ginsenoside 20 (S)-Rg3 on the progression of renal damage and dysfunction in type 2 diabetic Otsuka Long-Evans Tokushima Fatty rats," Biological \& Pharmaceutical Bulletin, vol. 33, no. 6, pp. 1077-1081, 2010.

[36] O. H. Lee, H. H. Lee, J. H. Kim et al., "Effect of ginsenosides Rg3 and Re on glucose transport in mature 3T3-L1 adipocytes," Phytotherapy Research, vol. 25, no. 5, 2011.

[37] T. Liu, Y. F. Peng, C. Jia et al., "Ginsenoside Rg3 improves erectile function in streptozotocin-induced diabetic rats," The Journal of Sexual Medicine, vol. 12, no. 3, pp. 611-620, 2015.

[38] D.-M. Lai, Y.-K. Tu, I.-M. Liu, P.-F. Chen, and J.-T. Cheng, "Mediation of $\beta$-endorphin by ginsenoside Rh2 to lower plasma glucose in streptozotocin-induced diabetic rats," Planta Medica, vol. 72, no. 1, pp. 9-13, 2006.

[39] L. Shih-Hsiang, H. Chao-Tien, N. Ho-Shan et al., "Ginsenoside Rh2 improves cardiac fibrosis via ppar $\delta$-STAT3 signaling in type 1-like diabetic rats," International Journal of Molecular Sciences, vol. 18, 2017.

[40] K.-T. Lee, T. W. Jung, H.-J. Lee, G.-S. Kim, Y. S. Shin, and W.-K. Whang, "The antidiabetic effect of ginsenoside Rb2 via activation of AMPK," Archives of Pharmacal Research, vol. 34, no. 7, pp. 1201-1208, 2011.

[41] S. Namgoong, H. Lee, S. K. Han, H. W. Lee, S. H. Jeong, and E. S. Dhong, "Effect of Panax ginseng extract on the activity of diabetic fibroblasts in vitro," International Wound Journal, vol. 16, no. 3, pp. 737-745, 2019.

[42] W.-b. Shang, C. Guo, J. Zhao et al., "Ginsenoside Rb1 upregulates expressions of GLUTs to promote glucose consumption in adiopcytes," Zhongguo Zhong Yao Za Zhi, vol. 39, pp. 4448-4452, 2014.

[43] Y. Wu, Z.-y. Xia, J. Dou et al., "Protective effect of ginsenoside $\mathrm{Rb} 1$ against myocardial ischemia/reperfusion injury in streptozotocin-induced diabetic rats," Molecular Biology Reports, vol. 38, no. 7, pp. 4327-4335, 2011.

[44] S. Wei, W. Li, Y. Yu et al., "Ginsenoside Compound K suppresses the hepatic gluconeogenesis via activating adenosine $-5^{\prime}$ monophosphate kinase: a study in vitro and in vivo," Life Sciences, vol. 139, pp. 8-15, 2015.
[45] N. Yang, P. Chen, Z. Tao et al., "Beneficial effects of ginsenoside-Rg1 on ischemia-induced angiogenesis in diabetic mice," Acta Biochimica et Biophysica Sinica, vol. 44, no. 12, pp. 999-1005, 2012.

[46] Y. Ying, Y.-1. Zhang, C.-j. Ma et al., "Neuroprotective effects of ginsenoside Rg1 against hyperphosphorylated tau-induced diabetic retinal neurodegeneration via activation of IRS-1/akt/gsk $3 \beta$ signaling," Journal of Agricultural and Food Chemistry, vol. 67, no. 30, pp. 8348-8360, 2019.

[47] J. Niu, Z. Pi, H. Yue, Y. Wang, Q. Yu, and S. Liu, "Effect of ginseng polysaccharide on the urinary excretion of type 2 diabetic rats studied by liquid chromatography-mass spectrometry," Journal of Chromatography B, vol. 907, pp. 7-12, 2012.

[48] H. Qian, S. Zhang, and Z. Shi, "Study on the mechanism of Panax ginseng polysaccharide on renal fibrosis of diabetic nephropathy through camp/PKA/CREB signal pathway," Chinese Pharmacological Bulletin, vol. 34, no. 5, pp. 695-701, 2018.

[49] X. Chang, K. Lu, L. Wang, M. Lv, and W. Fu, “Astraglaus polysaccharide protects diabetic cardiomyopathy by activating NRG1/ErbB pathway," BioScience Trends, vol. 12, no. 2, pp. 149-156, 2018.

[50] W. Chen, J. Ju, Y. Yang et al., "Astragalus polysaccharides protect cardiac stem and progenitor cells by the inhibition of oxidative stress-mediated apoptosis in diabetic hearts," Drug Design, Development and Therapy, vol. 12, pp. 943-954, 2018.

[51] W. Chen, Y. Lai, L. Xia et al., "Astragalus polysaccharides repress myocardial lipotoxicity in a PPARalpha-dependent manner in vitro and in vivo in mice," Journal of Diabetes and its Complications, vol. 29, no. 2, pp. 164-175, 2015.

[52] W. Chen, Y.-M. Li, and M.-H. Yu, "Astragalus polysaccharides: an effective treatment for diabetes prevention in NOD mice," Experimental and Clinical Endocrinology \& Diabetes, vol. 116, no. 8, pp. 468-474, 2008.

[53] W. Chen, Y.-M. Li, and M.-H. Yu, "Astragalus polysaccharides inhibited diabetic cardiomyopathy in hamsters depending on suppression of heart chymase activation," Journal of Diabetes and its Complications, vol. 24, no. 3, pp. 199-208, 2010.

[54] W. Chen, Q. Sun, J. Ju et al., "Effect of Astragalus polysaccharides on cardiac dysfunction in $\mathrm{db} / \mathrm{db}$ mice with respect to oxidant stress," BioMed Research International, vol. 2018, Article ID 8359013, 10 pages, 2018.

[55] S. Sun, S. Yang, M. Dai et al., "The effect of Astragalus polysaccharides on attenuation of diabetic cardiomyopathy through inhibiting the extrinsic and intrinsic apoptotic pathways in high glucose -stimulated H9C2 cells," $B M C$ Complementary and Alternative Medicine, vol. 17, p. 310, 2017.

[56] S. Tu, A. Shao, L. Ren et al., "Angiogenesis effect of Astragalus polysaccharide combined with endothelial progenitor cells therapy in diabetic male rat following experimental hind limb ischemia," Chinese Medical Journal, vol. 127, pp. 2121-2128, 2014.

[57] W. Chen, Y. Xia, X. Zhao et al., "The critical role of Astragalus polysaccharides for the improvement of PPAR $\alpha$ [correction of PPRA $\alpha$ ]-mediated lipotoxicity in diabetic cardiomyopathy," PLoS One, vol. 7, Article ID e45541, 2012.

[58] W. Chen, M.-H. Yu, Y.-M. Li, W.-J. Chen, and Y.-P. Xia, "Beneficial effects of astragalus polysaccharides treatment on cardiac chymase activities and cardiomyopathy in diabetic hamsters," Acta Diabetologica, vol. 47, no. S1, pp. 35-46, 2010. 
[59] S. Sun, S. Yang, N. An et al., "Astragalus polysaccharides inhibits cardiomyocyte apoptosis during diabetic cardiomyopathy via the endoplasmic reticulum stress pathway," Journal of Ethnopharmacology, vol. 238, p. 111857, 2019.

[60] N. Wang, D. Zhang, X. Mao, F. Zou, H. Jin, and J. Ouyang, "Astragalus polysaccharides decreased the expression of PTP1B through relieving ER stress induced activation of ATF6 in a rat model of type 2 diabetes," Molecular \& Cellular Endocrinology, vol. 307, no. 1-2, pp. 89-98, 2009.

[61] X. Q. Mao, Y. Wu, K. Wu et al., "Astragalus polysaccharide reduces hepatic endoplasmic reticulum stress and restores glucose homeostasis in a diabetic KKAy mouse model," Acta Pharmacologica Sinica, vol. 28, no. 12, pp. 1947-1956, 2007.

[62] G. Zhang, H. Fang, Y. Li et al., "Neuroprotective effect of Astragalus polysacharin on streptozotocin (STZ)-Induced diabetic rats," Medical Science Monitor, vol. 25, pp. 135-141, 2019.

[63] Li Cheng-De, Li Jing-Jing, L. Wang et al., "Inhibitory effect of Astragalus polysaccharides on apoptosis of pancreatic betacells mediated by Fas in diabetes mellitus rats," Zhong Yao Cai, vol. 34, pp. 1579-1582, 2011.

[64] M. Liu, J. Qin, H. Yarong et al., "Astragalus polysaccharide suppresses skeletal muscle myostatin expression in diabetes: involvement of ROS-ERK and NF- $\kappa \mathrm{B}$ pathways," Oxidative Medicine and Cellular Longevity, vol. 2013, Article ID 782497, 10 pages, 2013.

[65] J. Ju, W. Chen, Y. Lai et al., "Astragalus polysaccharides improve cardiomyopathy in STZ-induced diabetic mice and heterozygous (SOD2+/-) knockout mice," Brazilian Journal of Medical and Biological Research, vol. 50, p. e6204, 2017.

[66] C. Gu, Y. Zeng, Z. Tang et al., "Astragalus polysaccharides affect insulin resistance by regulating the hepatic SIRT1PGC- $1 \alpha /$ PPAR $\alpha$-FGF21 signaling pathway in male Sprague Dawley rats undergoing catch-up growth," Molecular Medicine Reports, vol. 12, no. 5, pp. 6451-6460, 2015.

[67] D. Gui, J. Huang, Y. Guo et al., "Astragaloside IV ameliorates renal injury in streptozotocin-induced diabetic rats through inhibiting NF- $\kappa \mathrm{B}$-mediated inflammatory genes expression," Cytokine, vol. 61, no. 3, pp. 970-977, 2013.

[68] B. Leng, F. Tang, M. Lu, Z. Zhang, H. Wang, and Y. Zhang, "Astragaloside IV improves vascular endothelial dysfunction by inhibiting the TLR4/NF- $\kappa \mathrm{B}$ signaling pathway," Life Sciences, vol. 209, pp. 111-121, 2018.

[69] K.-Q. He, W.-Z. Li, X.-Q. Chai, Y. Y. Yin, Y. Jiang, and W. P. Li, "Astragaloside IV prevents kidney injury caused by iatrogenic hyperinsulinemia in a streptozotocin-induced diabetic rat model," International Journal of Molecular Medicine, vol. 41, no. 2, pp. 1078-1088, 2017.

[70] Q. Nie, L. Zhu, L. Zhang, B. Leng, and H. Wang, “Astragaloside IV protects against hyperglycemia-induced vascular endothelial dysfunction by inhibiting oxidative stress and Calpain-1 activation," Life Sciences, vol. 232, p. 116662, 2019.

[71] R. Zhang, X. Zhang, B. Xing et al., "Astragaloside IV attenuates gestational diabetes mellitus via targeting NLRP3 inflammasome in genetic mice," Reproductive Biology and Endocrinology, vol. 17, p. 77, 2019.

[72] X. Zhang, M. Li, and H. Wang, "Astragaloside IV alleviates the myocardial damage induced by lipopolysaccharide via the toll-like receptor 4 (TLR4)/Nuclear factor kappa B (NF$\kappa \mathrm{B}) /$ Proliferator-Activated receptor $\alpha(\mathrm{PPAR} \alpha)$ signaling pathway," Medical Science Monitor, vol. 25, pp. 7158-7168, 2019.

[73] M. Ke, X.-Q. Hu, J. Ouyang, B. Dai, and Y. Xu, "The effect of astragalin on the VEGF production of cultured Müller cells under high glucose conditions," Bio-Medical Materials and Engineering, vol. 22, no. 1-3, pp. 113-119, 2012.

[74] L. Gao, Y.-W. Kwan, A. C. Bulmer, and C. W. K. Lai, "Noninvasive real-time characterization of renal clearance kinetics in diabetic mice after receiving danshensu treatment," Oxidative Medicine and Cellular Longevity, vol. 2018, Article ID 8267560, 10 pages, 2018.

[75] C. J. Jin, S. H. Yu, X. Wang et al., "The effect of lithospermic acid, an antioxidant, on development of diabetic retinopathy in spontaneously obese diabetic rats," PLoS One, vol. 9, Article ID e98232, 2014.

[76] E. S. Kang, G. T. Lee, B. S. Kim et al., "Lithospermic acid B ameliorates the development of diabetic nephropathy in OLETF rats," European Journal of Pharmacology, vol. 579, no. 1-3, pp. 418-425, 2008.

[77] S. H. Kim, S. H. Kim, M. Choi et al., "Natural therapeutic magnesium lithospermate B potently protects the endothelium from hyperglycaemia-induced dysfunction," Cardiovascular Research, vol. 87, no. 4, pp. 713-722, 2010.

[78] G. T. Lee, H. Ha, M. Jung et al., "Delayed treatment with lithospermate $\mathrm{B}$ attenuates experimental diabetic renal injury," Journal of the American Society of Nephrology, vol. 14, no. 3, pp. 709-720, 2003.

[79] M. Huang, P. Wang, S. Xu et al., "Biological activities of salvianolic acid B fromSalvia miltiorrhizaon type 2 diabetes induced by high-fat diet and streptozotocin," Pharmaceutical Biology, vol. 53, no. 7, pp. 1058-1065, 2015.

[80] S. Raoufi, T. Baluchnejadmojarad, M. Roghani, T. Ghazanfari, F. Khojasteh, and M. Mansouri, “Antidiabetic potential of salvianolic acid B in multiple low-dose streptozotocin-induced diabetes," Pharmaceutical Biology, vol. 53, no. 12, pp. 1803-1809, 2015.

[81] G. Qiang, X. Yang, L. Shi et al., "Antidiabetic effect of salvianolic acid $\mathrm{A}$ on diabetic animal models via AMPK activation and mitochondrial regulation," Cellular Physiology and Biochemistry, vol. 36, no. 1, pp. 395-408, 2015.

[82] Y. Ren, S. Tao, S. Zheng et al., "Salvianolic acid B improves vascular endothelial function in diabetic rats with blood glucose fluctuations via suppression of endothelial cell apoptosis," European Journal of Pharmacology, vol. 791, pp. 308-315, 2016.

[83] X.-Y. Yang, G.-F. Qiang, L. Zhang et al., "Salvianolic acid A protects against vascular endothelial dysfunction in high-fat diet fed and streptozotocin-induced diabetic rats," Journal of Asian Natural Products Research, vol. 13, no. 10, pp. 884-894, 2011.

[84] X.-y. Yang, L. Sun, P. Xu et al., "Effects of salvianolic scid A on plantar microcirculation and peripheral nerve function in diabetic rats," European Journal of Pharmacology, vol. 665, no. 1-3, pp. 40-46, 2011.

[85] Y. Xiaoyan, Z. Li, Y. Xiuying et al., "Salvianolic acid A protects the peripheral nerve function in diabetic rats through regulation of the AMPK-pgc1 $\alpha$-sirt3 Axis," Molecules, vol. 17, no. 9, pp. 11216-11228, 2012.

[86] X. Yu, L. Zhang, X. Yang et al., "Salvianolic acid A protects the peripheral nerve function in diabetic rats through regulation of the AMPK-PGC1 $\alpha$-sirt3 Axis," Molecules, vol. 17, no. 9, pp. 11216-11228, 2012.

[87] A. Arvindekar, T. More, P. V. Payghan, K. Laddha, N. Ghoshal, and A. Arvindekar, "Evaluation of anti-diabetic and alpha glucosidase inhibitory action of anthraquinones from Rheum emodi," Food \& Function, vol. 6, no. 8, pp. 2693-2700, 2015. 
[88] J. Xue, W. Ding, and Y. Liu, "Anti-diabetic effects of emodin involved in the activation of PPARgamma on high-fat dietfed and low dose of streptozotocin-induced diabetic mice," Fitoterapia, vol. 81, 2010.

[89] M. Xu, C. Hu, H.-h. Khan et al., "Argirein alleviates stressinduced and diabetic hypogonadism in rats via normalizing testis endothelin receptor A and connexin 43," Acta Pharmacologica Sinica, vol. 37, no. 2, pp. 246-254, 2016.

[90] K.-C. Lin, L.-R. Yeh, L.-J. Chen, Y.-J. Wen, K.-C. Cheng, and J.-T. Cheng, "Plasma glucose-lowering action of allantoin is induced by activation of imidazoline I-2 receptors in streptozotocin-induced diabetic rats," Hormone and Metabolic Research, vol. 44, no. 1, pp. 41-46, 2012.

[91] C.-S. Niu, W. Chen, H.-T. Wu et al., "Decrease of plasma glucose by allantoin, an active principle of yam (Dioscorea spp.), in streptozotocin-induced diabetic rats," Journal of Agricultural and Food Chemistry, vol. 58, no. 22, pp. 12031-12035, 2010.

[92] R. Huang, J. Xie, Y. Yu, and M. Shen, "Recent progress in the research of yam mucilage polysaccharides: isolation, structure and bioactivities," International Journal of Biological Macromolecules, 2019.

[93] B.-H. Lee, W.-H. Hsu, and T.-M. Pan, "Inhibitory effects of dioscorea polysaccharide on tnf- $\alpha$-induced insulin resistance in mouse fl83b cells," Journal of Agricultural and Food Chemistry, vol. 59, 2011.

[94] Z. Gao, R. Li, X. Lu, J. Liran, E. Huang, and J. Wang, "Effect of Chinese yam polysaccharide on renal function and aldose reductase pathway in diabetic nephropathy mice," Chinese Herbal Medicine, vol. 42, no. 3, pp. 643-646, 2019.

[95] H.-J. Choi, H.-J. Jang, T.-W. Chung et al., "Catalpol suppresses advanced glycation end-products-induced inflammatory responses through inhibition of reactive oxygen species in human monocytic THP-1 cells," Fitoterapia, vol. 86, pp. 19-28, 2013.

[96] C.-M. Lin, B.-W. Wang, W.-J. Fang, C.-M. Pan, K.-G. Shyu, and S.-W. Hou, "Catalpol ameliorates neointimal hyperplasia in diabetic rats," Planta Medica, vol. 85, no. 5, pp. 406-411, 2019.

[97] J. Liu, H.-R. Zhang, Y.-B. Hou, X.-L. Jing, X.-Y. Song, and X.-P. Shen, "Global gene expression analysis in liver of $\mathrm{db} / \mathrm{db}$ mice treated with catalpol," Chinese Journal of Natural Medicines, vol. 16, no. 8, pp. 590-598, 2018.

[98] J.-P. Shieh, K.-C. Cheng, H.-H. Chung, Y.-F. Kerh, C.-H. Yeh, and J.-T. Cheng, "Plasma glucose lowering mechanisms of catalpol, an active principle from roots of Rehmannia glutinosa, in streptozotocin-induced diabetic rats," Journal of Agricultural and Food Chemistry, vol. 59, no. 8, pp. 3747-3753, 2011.

[99] J. Yan, C. Wang, Y. Jin et al., "Catalpol ameliorates hepatic insulin resistance in type 2 diabetes through acting on AMPK/NOX4/PI3K/AKT pathway," Pharmacological Research, vol. 130, pp. 466-480, 2018.

[100] S. Yang, H. Deng, Q. Zhang et al., "Amelioration of diabetic mouse nephropathy by catalpol correlates with down-regulation of Grb10 expression and activation of insulin-like growth factor 1/insulin-like growth factor 1 receptor signaling," PLoS One, vol. 11, Article ID e0151857, 2016.

[101] B. Ying, R. Zhu, Y. Tian et al., "Catalpol in diabetes and its complications: a review of pharmacology, pharmacokinetics, and safety," Molecules, vol. 24, 2019.

[102] H. Zhang, Z.-M. Wu, Y.-P. Yang et al., "Catalpol ameliorates LPS-induced endometritis by inhibiting inflammation and
TLR4/NF- $\kappa \mathrm{B}$ signaling," Journal of Zhejiang UniversityScience B, vol. 20, no. 10, pp. 816-827, 2019.

[103] C. Yan, Q. Liu, Z. Shan et al., "The protective effect and mechanism of catalpol on high glucose-induced podocyte injury," BMC Complementary and Alternative Medicine, vol. 19, p. 244, 2019.

[104] G. Cao, H. Cai, B. Cai, and S. Tu, "Effect of 5-hydroxymethylfurfural derived from processed Cornus officinalis on the prevention of high glucose-induced oxidative stress in human umbilical vein endothelial cells and its mechanism," Food Chemistry, vol. 140, no. 1-2, pp. 273-279, 2013.

[105] Y. Chen, Y. Wu, X. Gan et al., "Iridoid glycoside from Cornus officinalis ameliorated diabetes mellitus-induced testicular damage in male rats: involvement of suppression of the AGEs/RAGE/p38 MAPK signaling pathway," Journal of Ethnopharmacology, vol. 194, pp. 850-860, 2016.

[106] W.-L. Jiang, S.-P. Zhang, J. Hou, and H.-B. Zhu, "Effect of loganin on experimental diabetic nephropathy," Phytomedicine, vol. 19, no. 3-4, pp. 217-222, 2012.

[107] C. H. Park, J. S. Noh, T. Tanaka, S. S. Roh, J. C. Lee, and T. Yokozawa, "Polyphenol isolated from Corni Fructus, 7-Ogalloyl-D-sedoheptulose, modulates advanced glycation endproduct-related pathway in type 2 diabetic $\mathrm{db} / \mathrm{db}$ mice," Archives of Pharmacal Research, vol. 38, no. 6, pp. 1270-1280, 2015.

[108] T. Yokozawa, K. S. Kang, C. H. Park et al., "Bioactive constituents of Corni Fructus: the therapeutic use of morroniside, loganin, and 7-O-galloyl-D-sedoheptulose as renoprotective agents in type 2 diabetes," Drug Discoveries \& Therapeutics, vol. 4, pp. 223-234, 2010.

[109] M.-Y. Qi, H.-R. Liu, D.-Z. Dai, N. Li, and Y. Dai, “Total triterpene acids, active ingredients from Fructus Corni, attenuate diabetic cardiomyopathy by normalizing ET pathway and expression of FKBP12.6 and SERCA2a in streptozotocin-rats," Journal of Pharmacy and Pharmacology, vol. 60, no. 12, pp. 1687-1694, 2008.

[110] H.-Q. Xu and H.-P. Hao, "Effects of iridoid total glycoside from cornus officinalis on prevention of glomerular overexpression of transforming growth factor beta 1 and matrixes in an experimental diabetes model," Biological \& Pharmaceutical Bulletin, vol. 27, no. 7, pp. 1014-1018, 2004. 